\title{
Revaluing the Role of vmPFC in the Acquisition of Pavlovian Threat Conditioning in Humans
}

\author{
${ }^{\circledR}$ Simone Battaglia, ${ }^{\circledR}$ Sara Garofalo, ${ }^{\circledR}$ Giuseppe di Pellegrino, and ${ }^{\circledR}$ Francesca Starita \\ Center for Studies and Research in Cognitive Neuroscience, Department of Psychology, University of Bologna, 40126 Bologna, Italy
}

The role of the ventromedial prefrontal cortex (vmPFC) in human pavlovian threat conditioning has been relegated largely to the extinction or reversal of previously acquired stimulus-outcome associations. However, recent neuroimaging evidence questions this view by also showing activity in the vmPFC during threat acquisition. Here we investigate the casual role of vmPFC in the acquisition of pavlovian threat conditioning by assessing skin conductance response (SCR) and declarative memory of stimulus-outcome contingencies during a differential pavlovian threat-conditioning paradigm in eight patients with a bilateral vmPFC lesion, 10 with a lesion outside PFC and 10 healthy participants (each group included both females and males). Results showed that patients with vmPFC lesion failed to produce a conditioned SCR during threat acquisition, despite no evidence of compromised SCR to unconditioned stimulus or compromised declarative memory for stimulus-outcome contingencies. These results suggest that the vmPFC plays a causal role in the acquisition of new learning and not just in the extinction or reversal of previously acquired learning, as previously thought. Given the role of the vmPFC in schemarelated processing and latent structure learning, the vmPFC may be required to construct a detailed representation of the task, which is needed to produce a sustained conditioned physiological response in anticipation of the unconditioned stimulus during threat acquisition.

Key words: aversive learning; fear conditioning; latent structure learning; schema processing; threat acquisition; ventromedial prefrontal cortex

Significance Statement

Pavlovian threat conditioning is an adaptive mechanism through which organisms learn to avoid potential threats, thus increasing their chances of survival. Understanding what brain regions contribute to such a process is crucial to understand the mechanisms underlying adaptive as well as maladaptive learning, and has the potential to inform the treatment of anxiety disorders. Importantly, the role of the ventromedial prefrontal cortex (vmPFC) in the acquisition of pavlovian threat conditioning has been relegated largely to the inhibition of previously acquired learning. Here, we show that the vmPFC actually plays a causal role in the acquisition of pavlovian threat conditioning.

\section{Introduction}

Extensive research shows the involvement of the human ventromedial prefrontal cortex (vmPFC) in the extinction and reversal of pavlovian threat conditioning (Morris and Dolan, 2004; Phelps et al., 2004; Kalisch et al., 2006; Sotres-Bayon et al., 2006; Milad et al., 2007; Quirk and Mueller, 2008; Schiller et al., 2008; Schiller and Delgado, 2010; Hartley et al., 2011; Milad and

Received Feb. 10, 2020; revised July 22, 2020; accepted Aug. 5, 2020.

Author contributions: S.B. and G.d.P. designed research; S.B. performed research; S.B., S.G., and F.S. analyzed data; F.S. wrote the paper.

This work was supported by RF0 Grant from the University of Bologna awarded to G.d.P. We thank Prof. Elisa Ciaramelli for comments during manuscript preparation. We also thank Jacopo Straccialini for his assistance with data collection; Giulia Petrillo, Giulio Palmas, and Davide Braghittoni for the neuropsychological assessment; and Prof. Caterina Bertini and Flavia de Luca for support with lesion analysis.

The authors declare no competing financial interests.

Correspondence should be addressed to Giuseppe di Pellegrino at g.dipellegrino@unibo.it.

https://doi.org/10.1523/JNEUROSCI.0304-20.2020

Copyright $\odot 2020$ the authors
Quirk, 2012; Raij et al., 2018; Dunsmoor et al., 2019; Savage et al., 2020). In particular, this region may not simply inhibit the expression of amygdala-dependent conditioned threat response, but signal a change in previously acquired contingencies to select the most appropriate response to the current situation (Bukalo et al., 2015; Do-Monte et al., 2015; Delgado et al., 2016; Dunsmoor et al., 2019). Crucially, recent neuroimaging evidence questions this view by showing activity in the vmPFC also during threat acquisition (Fullana et al., 2016; Harrison et al., 2017; Dunsmoor et al., 2019), also raising the hypothesis for a role of this region during the initial acquisition of new learning.

Beyond the literature on threat conditioning, the vmPFC has a crucial role in value and stimulus-outcome representation (Schoenbaum et al., 2009, 2011; Hiser and Koenigs, 2018), model-based computations (Wilson et al., 2014), and schemarelated processing and latent structure learning (Gershman and Niv, 2010; Gershman et al., 2015; Niv, 2019; Schuck et al., 2019). In fact, all these processes appear involved during the acquisition 
Table 1. Demographic characteristics and neuropsychological assessment for all groups

\begin{tabular}{|c|c|c|c|c|c|c|c|}
\hline & $\begin{array}{l}\text { vmPFC } \\
(n=8)\end{array}$ & $\begin{array}{l}\mathrm{BDC} \\
(n=10)\end{array}$ & $\begin{array}{l}\mathrm{HC} \\
(n=10)\end{array}$ & Test & $\mathrm{df}$ & $p$ & $\eta_{p}^{2}$ \\
\hline \multicolumn{8}{|l|}{ Demographics } \\
\hline Age (years) & $57.25 \pm 6.25$ & $51.10 \pm 14.37$ & $67.90 \pm 7.87$ & $F=6.69$ & 2,25 & $0.005^{+}$ & 0.34 \\
\hline Education (years) & $10.5 \pm 2.67$ & $13.30 \pm 4.37$ & $13.00 \pm 2.45$ & $F=1.83$ & 2,25 & 0.18 & 0.12 \\
\hline Chronicity (years) & $5.87 \pm 4.94$ & $2.80 \pm 2.20$ & & $F=3.13$ & 1,16 & 0.09 & 0.16 \\
\hline $\operatorname{Sex}(M / F)$ & $6 / 2$ & $6 / 4$ & $4 / 6$ & $\chi^{2}=1.27$ & 2 & 0.32 & \\
\hline \multicolumn{8}{|l|}{ Neuropsychological assessment } \\
\hline Raven Progressive Matrices* & $3.05 \pm 0.75$ & $3 \pm 1.09$ & & 1.02 & 1,12 & 0.33 & 0.07 \\
\hline Stroop Test* & $2.25 \pm 1.91$ & $2.5 \pm 1.04$ & & 0.08 & 1,12 & 0.77 & 0.007 \\
\hline Towers of London\# & $99.14 \pm 16.40$ & $104 \pm 13.56$ & & 0.33 & 1,11 & 0.57 & 0.02 \\
\hline Digit Span* & $3.75 \pm 0.46$ & $3.16 \pm 1.60$ & & 0.97 & 1,12 & 0.34 & 0.07 \\
\hline Phonemic Fluency* & $3.25 \pm 1.16$ & $3.16 \pm 1.32$ & & 0.02 & 1,12 & 0.90 & 0.001 \\
\hline Semantic Fluency* & $3.75 \pm 0.74$ & $3.66 \pm 0.81$ & & 0.48 & 1,12 & 0.49 & 0.03 \\
\hline
\end{tabular}

All measures are reported as mean $\pm S D$, except for Sex, which is reported as frequency. M, Male; F, female.

*Equivalent score.

\#Standard score.

${ }^{+}$Post hoc analysis reported a significant difference between BDC and HC groups, $p=0.01$ and vmPFC group versus $\mathrm{HC}$ group, $p=0.02$; vmPFC group versus $\mathrm{BDC}$ group was not significant $p=0.8$.

of pavlovian conditioning, which consists in updating the value of encountered stimuli based on changes in stimulus-outcome contingencies. Additionally, cognitive and computational models have traditionally described this updating in terms of model-free mechanisms, in which prediction errors following the unexpected occurrence of unconditioned stimuli (USs) drive the increment of predictions for conditioned stimuli (CSs; Rescorla and Wagner, 1972; Sutton, 1988). Nevertheless, recent evidence shows the recruitment of model-based mechanisms to represent pavlovian contingencies (Prévost et al., 2013; Pauli et al., 2019), suggesting that an abstract representation of the broader structure of the pavlovian contingencies is also taken into account when computing predictions for CS, at least in humans.

The neglected role of vmPFC in threat acquisition may have been reinforced also by a lack of neuropsychological studies assessing the consequences of a lesion to the vmPFC on the acquisition of threat conditioning. In fact, the only existing study on patients with vmPFC lesions found preserved conditioned skin conductance response (SCR) to the presentation of an image previously associated with an aversive sound $\left(\mathrm{CS}^{+}\right)$, despite a failure to show anticipatory SCR to negative (and positive) outcomes during a gambling task (Bechara et al., 1999). Nevertheless, this evidence should be taken cautiously because of the limited sample size $(n=5)$ and the diverse etiology of the lesion among patients (meningioma, stroke, and cyst). Additionally, the acquisition of conditioning was evaluated in terms of an increase in SCR to the $\mathrm{CS}^{+}$during acquisition compared with habituation and extinction, rather than comparing the SCR elicited by the $\mathrm{CS}^{+}$during each phase to that elicited by a control stimulus never associated with an aversive sound $\left(\mathrm{CS}^{-}\right)$. In fact, given that the putative role in discriminating threat from safety (Fullana et al., 2016), the impairment in the acquisition of threat conditioning in patients with $\mathrm{vmPFC}$ lesions may consist in a failure in generating differential SCR between $\mathrm{CS}^{+}$and $\mathrm{CS}^{-}$, rather than an overall failure in increasing SCR to a $\mathrm{CS}^{+}$during acquisition.

The present study aims to reevaluate the role of the vmPFC in the acquisition of pavlovian threat conditioning. Eight patients with a naturally occurring bilateral lesion to the vmPFC and adjacent perigenual tissue, a group of healthy participants and a group of patients with a lesion outside PFC completed a differential threat conditioning paradigm, while their SCR to $\mathrm{CS}^{+}, \mathrm{CS}^{-}$, and US (aversive electrical stimulation) was recorded. Explicit awareness of CS-US contingencies was also assessed.

\section{Materials and Methods}

Participants

Thirty right-handed adults participated in the study, equally divided into three groups, namely patients with a lesion affecting the vmPFC (vmPFC patients), brain-damaged control patients (BDCs) with a lesion that did not involve the PFC, and healthy control participants (HCs; Table 1, demographic and clinical information). The vmPFC patients were eligible for inclusion in the present study if their mapped lesion was centered on the vmPFC (i.e., the majority of lesioned tissue must pertain to the vmPFC), defined as the medial one-third of the orbital surface and the ventral one-third of the medial surface of prefrontal cortex, bilaterally. Data were initially collected on $10 \mathrm{vmPFC}$ patients, but 2 patients were subsequently found to have lesions extending to the medial temporal lobe and/or insula, and so were excluded from the analysis. For all patients, lesions were naturally occurring, acquired in adulthood, and were stable since the patient's most recent neuroimaging session and corresponding lesion analysis (see below). Participants with a history of progressive disease, psychiatric disease, drug or alcohol abuse requiring treatment, premorbid head injury, premorbid/comorbid neurologic disease, severe aphasia, visual neglect, or marked sensory impairment were excluded from participation. Groups were matched in terms of sex, education, illness chronicity, and neuropsychological assessment scores (Table 1). Groups differed in terms of age; therefore, this variable was used as covariate in all analyses (Table 1).

Participants were recruited at the Center for Studies and Research in Cognitive Neuroscience of Bologna University, where the study was conducted. They had normal or corrected-to-normal vision and were naive to the purposes of the study. All participants gave informed written consent to participation after being informed about the procedure of the study. The study was conducted in accordance with the ethical principles of the World Medical Association Declaration of Helsinki and approved by the Bioethics Committee of the University of Bologna.

\section{Neuropsychological assessment}

vmPFC and BDC patients were given a series of standardized neuropsychological tests. The selected neuropsychological battery included the following tests: Raven Progressive Matrices (Spinnler and Tognoni, 1987), Stroop Test (Caffarra et al., 2002), Tower of London (Kennedy et al., 2000; the total number of correct moves was used for analysis), Digit Span (Orsini et al., 1987), and Phonemic and Semantic Fluency (Novelli et al., 1986). Normative scores derived from a nationally representative sample of adults are available for each test. For Tower of London, patients' raw scores were converted in Standard scores (Culbertson and Zillmer, 1999). For all the other tests, patients' raw scores were converted into equivalent scores (Capitani and Laiacona, 1988), adjusted for age and years of education. Equivalent score is a 5 point scale, ranging from 0 to 4 , with $0=$ pathologic performance, $1=$ borderline performance, and 
2-4 = normal performance. $t$ Tests in Table 1 showed no significant difference between patients with a lesion affecting the vmPFC and BDC on any of the tests (all $p>0.25$ ).

\section{Lesion analysis}

Lesion etiologies for the vmPFC group were aneurysm of the anterior communicating artery $(n=7)$ and aneurysm of the anterior cerebral artery $(n=1)$. Lesion etiologies for the BDC group were meningioma resection $(n=2)$, arteriovenous malformation resection $(n=1)$, cerebrovascular accidents $(n=5)$, and abscess $(n=2)$.

For the vmPFC group, imaging of lesions was available for all patients, while for the BDC group imaging of lesions was available for 8 of 10 patients. For each patient, lesion extent and location were documented using the most recent clinical computed tomography $(n=5 \mathrm{vmPFC}$ patients; $n=1 \mathrm{BDCs}$ ) or magnetic resonance imaging (MRI; $n=3 \mathrm{vmPFC}$ patients; $n=7 \mathrm{BDC}$ ). Lesions were traced directly on each slice of the normalized T1-weighted template MRI scan from the Montreal Neurologic Institute (Holmes et al., 1998) by a trained neuropsychologist (S.B.) and checked by a neurologist (G.d.P.). This template is approximately oriented to match Talairach space and distributed with MRIcro (Holmes et al., 1998). MRIcro (Rorden and Brett, 2000) was used to estimate lesion volume (in $\mathrm{cm}^{3}$ ) and generate brain lesion overlap images.

Figure 1 shows the extent of individual brain lesions and overlap of brain lesions in vmPFC patients. In the vmPFC group, the Brodmann's areas (BAs) that were mainly affected were BAs 10, 11, 24, 25, and 32. The region of maximal overlap occurred in BA 11 (mean $=16.55 \mathrm{cc}$, $\mathrm{SD}=9.75 \mathrm{cc})$, and BA $10($ mean $=8.09 \mathrm{cc}, \mathrm{SD}=7.92 \mathrm{cc})$. This region of maximal overlap plays a causal role in the psychophysiological and behavioral outcomes under investigation (Koenigs et al., 2007). Nevertheless, although BAs 24 and 32 were not included in the region of maximal overlap, a recent meta-analysis reported correlational evidence of the involvement of the dorsal portion of these BAs [i.e., dorsal anterior cingulate cortex (dACC)] during the acquisition of threat conditioning (Fullana et al., 2016). Thus, we decided to conduct a detailed quantification of the extent of the damage to the dACC and to the vmPFC per se, and their relative contribution to the entire lesion. To this end, we used MRIcro (Rorden and Brett, 2000) to define dACC and vmPFC regions of interest (ROIs). The dACC ROI included the dorsal BAs 24 and 32 (as defined by Bush et al., 2000), while the vmPFC ROI included BAs 10, 11, and 25 and the rostral BAs 24 and 32 (as defined by Bush et al., 2000). In none of the patients whose lesion touched the dACC, the dACC damage extended to the coordinates reported in the metanalysis by Fullana et al. (2016) as active during threat acquisition. Additionally, such damage represented on average only $4.27 \%$ ( minimum $=0.06 \%$, maximum $=11.73 \%)$ of the entire lesion. In contrast, the vmPFC damage represented on average $88.66 \%$ (minimum $=$ $73.30 \%$, maximum $=98.78 \%$ ) of the entire lesion. Additionally, the percentage of vmPFC damage relative to the entire lesion was greater than the percentage of dACC damage (paired $t$ test, $p<0.001$ ). Thus, we can confirm that, in our patients, lesions are centered on the vmPFC, with the majority of lesioned tissue pertaining selectively to this region.

In the BDC group, the BAs that were mainly affected were BA 17,18 , and 19 (mean $=8.19 \mathrm{cc}, \mathrm{SD}=11.77 \mathrm{cc}$ ); BA 20, 21, 22, and 37 (mean = $16.80 \mathrm{cc}, \mathrm{SD}=23.12 \mathrm{cc}$ ); and BA 39 and 40 (mean $=2.07 \mathrm{cc}, \mathrm{SD}=$ $3.76 \mathrm{cc}$ ). There was no significant difference in lesion volume between vmPFC patients and BDCs $\left(\operatorname{mean}_{\mathrm{vmPFC}}=36.76 \mathrm{cc}, \operatorname{mean}_{\mathrm{BDC}}=46.32 \mathrm{cc}\right.$; $t$ test: $t=0.60, p=0.557$ ). For the BDCs whose brain images were not available, lesion affected the left calcarine sulcus and adjacent regions in one case, and the region proximal to the temporal horn of the left lateral ventricle in the other case, as reported in their medical records.

\section{Pavlovian threat conditioning task}

The CSs were images of two different objects (i.e., a lamp and a plant), which appeared embedded in an indoor scene for $4 \mathrm{~s}$ (previously used in Battaglia et al., 2018). Their presentation was followed by a jittered $14-17 \mathrm{~s}$ intertrial interval, consisting of a gray screen. The trial order was pseudorandomized, such that no more than three identical CSs occurred in a row. To reduce initial orienting responses, the experiment began with a short habituation phase, which included two presentations of each CS (data not analyzed). Then acquisition included 40 trials $\left(20 \mathrm{CS}^{+}, 20 \mathrm{CS}^{-}\right)$.
Presentation of the threat conditioned stimulus $\left(\mathrm{CS}^{+}\right.$, object assignment counterbalanced between participants) coterminated with the delivery of the aversive electrical shock (i.e., US) in 12 of 20 trials (60\% reinforcement rate). The shock was generated by a Digitimer Stimulator (model DS7, Digitimer) and delivered to the participants' left inner wrist through pregelled snapped electrodes. Presentation of the other object was never paired with the US, representing the within-subject control stimulus $\left(\mathrm{CS}^{-}\right)$. Acquisition always started with a presentation of a $\mathrm{CS}^{-}$and of a reinforced $\mathrm{CS}^{+}$, in random order. Extinction included 40 trials $\left(20 \mathrm{CS}^{+}, 20 \mathrm{CS}^{-}\right)$, during which CS presentation was never paired with the US.

\section{Dependent variables}

Skin conductance response. SCR was recorded continuously during the task from two $\mathrm{Ag} / \mathrm{AgCl}$ electrodes (model TSD203, BIOPAC Systems) filled with isotonic hyposaturated conductant and attached to the distal phalanges of the second and the third finger of participants' left hands. The signal was amplified with a DC amplifier (model EDA100C, BIOPAC Systems) with a gain factor of $5 \mu \mathrm{S} / \mathrm{V}$ and a lowpass filter of $10 \mathrm{~Hz}$. The analog signal was digitalized at $200 \mathrm{~Hz}$ using a BIOPAC Systems MP-150 digital converter and fed into AcqKnowledge 3.9 (BIOPAC Systems) for offline analysis.

The digitalized signal was analyzed using custom-made MATLAB scripts to obtain amplitude SCR values, operationalized as the peak-topeak amplitude of the largest deflection occurring between 0.5 and $4.5 \mathrm{~s}$ after event onset. The minimum response criterion was $0.02 \mu \mathrm{S}$, and smaller responses were encoded as 0 . Raw SCR scores were square root transformed to normalize the data distribution (Siddle et al., 1988). SCR amplitude following the CS was analyzed to assess conditioned learning. Stimulus onset referred to the time of CS appearance on the screen. To reduce interindividual variability, SCR was scaled to each participant's mean square root-transformed US response (Schiller et al., 2008; see below for its computation). Thus, raw scores were range corrected by dividing each individual score by the participants mean SCR to US (Lykken, 1972). This procedure can reduce error variance, thus increasing statistical power when comparing groups of participants. In this way, conditioned responses can be directly compared across groups without confounding baseline differences in skin conductance levels (LaBar et al., 2004). To assess conditioned responses to the $\mathrm{CS}^{+}$, only nonreinforced $\mathrm{CS}^{+}$trials were analyzed $(n=8)$ to exclude any confounding effect of shock on SCR. SCR amplitude following the US was analyzed to assess the unconditioned response. Stimulus onset was represented by the time of shock administration, mean SCRs to the 12 shocks were analyzed. Additionally, to further evaluate the unconditioned response, we computed and analyzed SCR latency following US administration (i.e., the temporal interval between shock administration and SCR onset; Sjouwerman and Lonsdorf, 2019).

Explicit contingency awareness. To assess the explicit awareness of CS-US contingencies, at the end of the conditioning task participants were presented with one CS at the time and asked to indicate whether or not the stimulus was associated with a shock during the task. A score of 1 was given for a correct answer and a score of 0 for an incorrect answer.

\section{Procedure}

Participants were seated comfortably in a silent and dimly lit room, and their position was centered, relative to the computer screen, at a $100 \mathrm{~cm}$ viewing distance. Electrodes for SCR recording and for shock administration were attached to the participant. After verifying the correct recording of SCR, shock intensity was adjusted using a standard workup procedure. It was initially set at $0.5 \mathrm{~mA}$ and increased by $1 \mathrm{~mA}$ until participants reported it as "highly uncomfortable, but not painful." Participants were asked to remain as quiet and still as possible during the task and to keep their attention on the center of the screen. At the beginning of the conditioning task, participants were informed that different visual stimuli would appear on the screen, and some of them might be paired with the shock. Participants received the instruction to carefully observe and learn which stimuli were paired with the shock. Participants were also informed that their behavior had no effect on shock delivery. At the end of the conditioning task, CS-US contingency awareness was tested. 


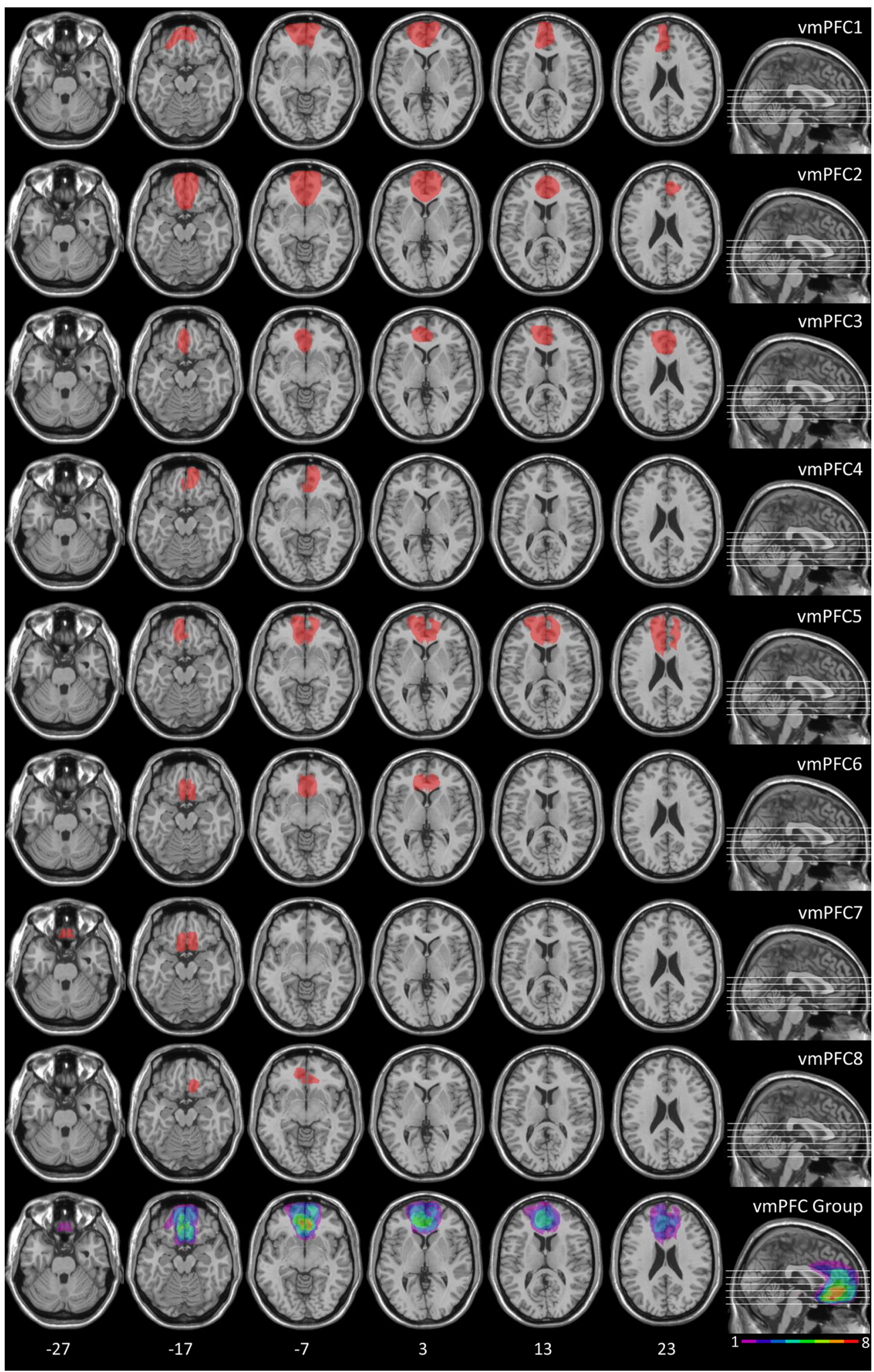

Figure 1. Location and overlap of brain lesions of vmPFC patients. Representative axial slices showing individual patients' lesions, and representative axial slices and midsagittal view showing cumulative lesions overlap. The white horizontal lines on the sagittal view indicate the positions of the axial slices. The white numbers below the axial slices indicate the $z$-coordinates of each slice. The color bar indicates the number of overlapping lesions. The left hemisphere is on the left side. Maximal overlap occurs in BAs 10 and 11.

Experimental design and statistical analysis

Statistical analyses were performed using JASP (version 0.11.1; JASP Team 2019). Assumptions for a correct use of parametric analyses were assessed. The significance threshold was $p<0.05$, and post hoc analyses were conducted with Tukey's HSD test. Reported $p$ values are always intended as two tailed. Since BDCs and HCs differed in terms of age (Table 1), this variable was used as a covariate in all ANOVAs. In all analyses, the covariate age did not have any statistically significant influence (all $p \geq 0.154$ ). Cumming estimation plots (Ho et al., 2019) were used to illustrate the data, whenever appropriate. 

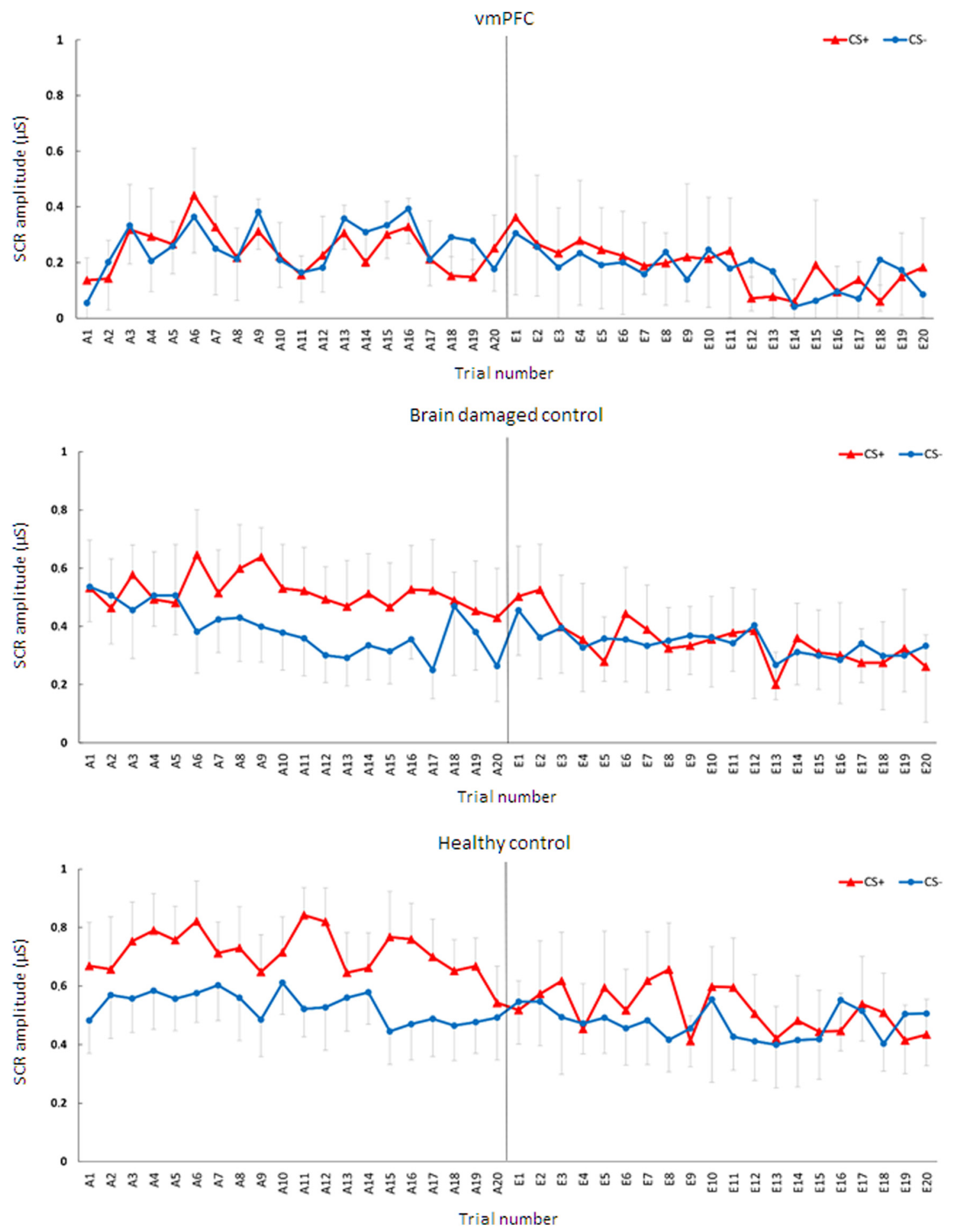

Figure 2. Trial by trial mean $\mathrm{SCR}$ to $\mathrm{CS}^{+}$and $\mathrm{CS}^{-}$during acquisition and extinction for the three groups. Error bars represent the $95 \%$ confidence interval.

Voxel-based lesion symptom mapping analysis

To gather further evidence regarding the location of the lesioned voxels contributing to the reduced differential SCR during acquisition in vmPFC patients, we conducted a voxel-based lesion symptom-mapping analysis using the Non-Parametric Mapping software (Rorden et al., 2007). Traced lesions of the vmPFC and BDC groups, and differential SCRs between $\mathrm{CS}^{+}$and $\mathrm{CS}^{-}$were used to compare performances of patients with and without damage at each voxel in the brain. Voxels were only included in the analysis if $\geq 20 \%$ patients had nonzero lesion values in that voxel. The nonparametric Brunner-Munzel (BM) rankorder test (Brunner and Munzel, 2000) was used with $\alpha$ set at $p<0.05$,
1000 permutations, resulting in a critical $z$ value of 2.92 . The threshold for the extent of the cluster of voxel was set at 50 voxels (Gläscher et al., 2010).

\section{Results}

Figure 2 shows a trial-by-trial representation of the mean SCR to $\mathrm{CS}^{+}$and $\mathrm{CS}^{-}$during acquisition and extinction in the three experimental groups. Qualitatively, observing the figure, the healthy control and brain damage control groups show an increase in mean SCR to $\mathrm{CS}^{+}$relative to $\mathrm{CS}^{-}$over the course of 
A
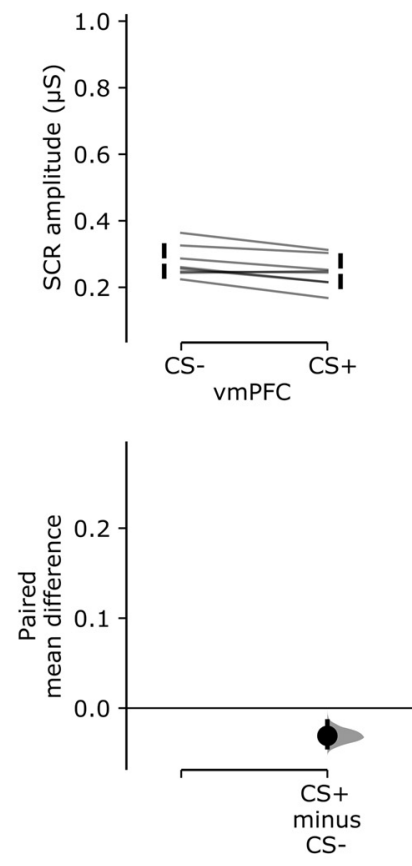
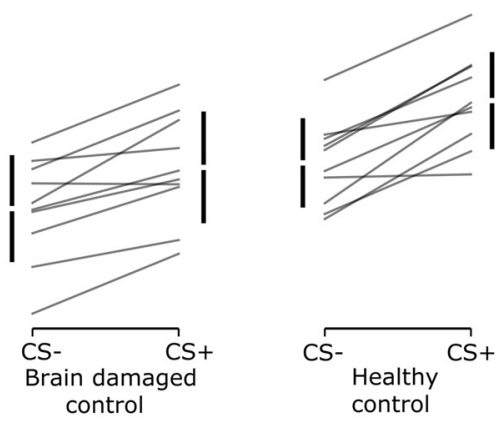
control

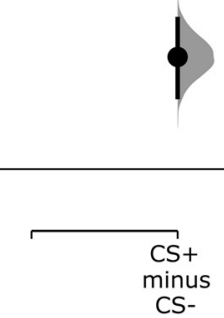

B
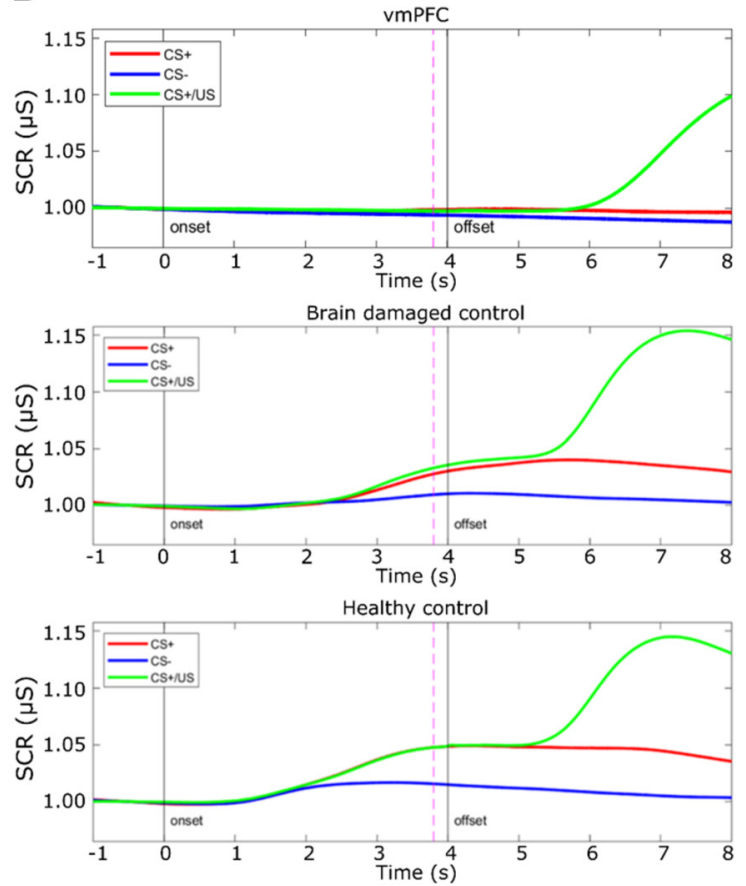

Figure 3. Acquisition of threat conditioning. A, Cumming estimation plots showing raw data and paired mean difference of SCR amplitude to $\mathrm{CS}^{+}$and $\mathrm{CS}^{-}$for the vmPFC group, the braindamaged control group, and the healthy control group. On the top axes, each paired set of observations is connected by a line. Also, 95\% confidence intervals are indicated by vertical error bars, and group means are depicted as white gaps between the bars. On the lower axes, $95 \%$ confidence intervals are indicated by vertical error bars, and mean differences, plotted as a bootstrap sampling distribution (5000 samples), are depicted as dots. B, Grand average event-related SCR illustrates mean SCR over time to $\mathrm{CS}^{+}$(no shock trials), $\mathrm{CS}^{-}$and $C S^{+} / \mathrm{US}$ (shock trials). SCR is expressed as a proportion of the baseline (1 $\mathrm{s}$ before $C S$ onset). Continuous vertical lines indicate the time of onset and offset of the CSs. Dashed line indicates shock onset in CS ${ }^{+} / U S$ trials.

acquisition, which then fades over the course of extinction. In contrast, in vmPFC no increase in mean SCR to $\mathrm{CS}^{+}$trials appears evident during any of the acquisition trials.

\section{Acquisition}

First, we assessed whether lesion to vmPFC affects threat acquisition with a $2 \times 3$ mixed design ANCOVA (stimulus, $\mathrm{CS}^{+} / \mathrm{CS}^{-}$; group, vmPFC/BDC/HC; age as covariate; see Materials and Methods for more details). There was no significant main effect of stimulus $(p=0.229)$. Instead, there was a significant main effect of group $\left[F_{(2,24)}=16.25 ; p<0.001 ; \eta_{\mathrm{p}}^{2}=0.57 ; 95 \%\right.$ confidence interval (CI), 0.25, 0.71], qualified by a significant stimulus by group interaction $\left(F_{(2,24)}=17.49 ; p<0.001 ; \eta_{\mathrm{p}}^{2}=0.59 ; 95 \%\right.$ $\mathrm{CI}, 0.27,0.72)$. HCs and BDCs responded more to $\mathrm{CS}^{+}$than to $\mathrm{CS}^{-}$(HCs: $\mathrm{CS}^{+}$, mean $=0.72 \mu \mathrm{S} ; \mathrm{CS}^{-}$, mean $=0.53 \mu \mathrm{S} ; \mathrm{p}<$ 0.001 ; BDCs: $\mathrm{CS}^{+}$, mean $=0.52 \mu \mathrm{S}$; $\mathrm{CS}^{-}$, mean $=0.39 \mu \mathrm{S}$; $p<0.001)$. In contrast, vmPFC did not show any significant difference in response between $\mathrm{CS}^{+}$and $\mathrm{CS}^{-}\left(\mathrm{CS}^{+}\right.$, mean $=0.25$ $\mu \mathrm{S}$; $\mathrm{CS}^{-}$, mean $=0.27 \mu \mathrm{S} ; p=0.965$; Fig. $3 A$ ). Thus, while HCs and BDCs showed successful acquisition of threat conditioning, vmPFC patients were impaired in the acquisition of threat conditioning. Also, observing Figure $3 B$, following CS presentation, the healthy control and brain-damaged control groups show an increase in SCR to $\mathrm{CS}^{+}$and $\mathrm{CS}^{+} / \mathrm{US}$ compared with $\mathrm{CS}^{-}$as well as an increase following US delivery. In contrast, the vmPFC group shows only an increase in SCR following US delivery.

\section{Extinction}

During extinction, the $2 \times 3$ mixed-design ANCOVA (stimulus, $\mathrm{CS}^{+} / \mathrm{CS}^{-}$; group, vmPFC/BDC/HC; age as covariate) showed only a main effect of group $\left(F_{(2,24)}=5.57 ; p=0.01 ; \eta_{\mathrm{p}}^{2}=0.32\right.$; $95 \% \mathrm{CI}, 0.02,0.52)$. vmPFC patients responded significantly less than HCs (vmPFC patients, mean $=0.19 \mu \mathrm{S}$; control participants, mean $=0.49 \mu S ; p=0.001$ ) but not less than BDCs (BDCs, mean $=0.34 \mu \mathrm{S} ; p=0.119)$. There was no significant difference between HCs and BDCs $(p=0.115)$. No other main effects or interactions were significant (all $p \geq 0.802$ ). Therefore, HCs and BDCs had successful extinction of threat conditioning.

\section{US intensity, SCR to the US, and SCR latency to the US}

We then tested the possibility that differences in threat acquisition between groups may have resulted from differences in the intensity of delivered US, SCR to the US, and latency of the SCR. The one-way ANCOVA (group, vmPFC/BDC/HC; age as covariate) on mean US intensity showed no significant effect of group $\left(F_{(2,24)}=1.81 ; p=0.185 ; \eta_{\mathrm{p}}^{2}=0.13 ; 95 \% \mathrm{CI}, 0.00,0.34\right.$; vmPFC patients, mean $=6.15 \mathrm{~mA}$; BDCs, mean $=7.44 \mathrm{~mA}$; HCs, mean $=$ $7.95 \mathrm{~mA}$ ). Similarly, the one-way ANCOVA (group, vmPFC/ $\mathrm{BDC} / \mathrm{HC}$; age as covariate) on mean SCR to the US showed no significant effect of group $\left(F_{(2,24)}=0.91 ; p=0.414 ; \eta_{\mathrm{p}}^{2}=0.07\right.$; $95 \% \mathrm{CI}, 0.00,0.26$; vmPFC patients, mean $=0.86 \mu \mathrm{S}$; BDCs, mean $=1.05 \mu \mathrm{S}$; HCs, mean $=1.01 \mu \mathrm{S})$. Importantly, all participants exhibited a reliable SCR to the US. Additionally, it is worth noting that even in four vmPFC patients who had mean SCRs to the US of $1.00,1.05,1.07$, and $1.32 \mu \mathrm{S}$, the difference in SCR between $\mathrm{CS}^{+}$and $\mathrm{CS}^{-}$was $-0.05,-0.00,-0.02$, and $0.00 \mu \mathrm{S}$, respectively, suggesting no evidence of threat acquisition, despite SCR to the US similar to or greater than the mean SCR of healthy participants.

Finally, the one-way ANCOVA (group, vmPFC/BDC/HC; age as covariate) on SCR latency to the US showed a significant effect of group $\left(F_{(2,24)}=4.31 ; p=0.025 ; \eta_{\mathrm{p}}^{2}=0.26 ; 95 \% \mathrm{CI}, 0.00\right.$, 0.47; Fig. $4 C)$. SCR latency of vmPFC patients $($ mean $=2.03 \mathrm{~s})$ was greater than that of BDCs $($ mean $=1.06 \mathrm{~s}, p=0.014)$. In 
A
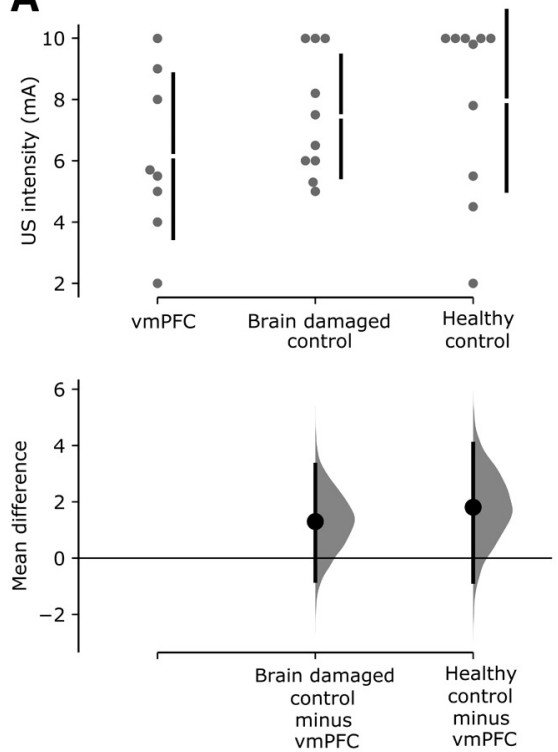

B
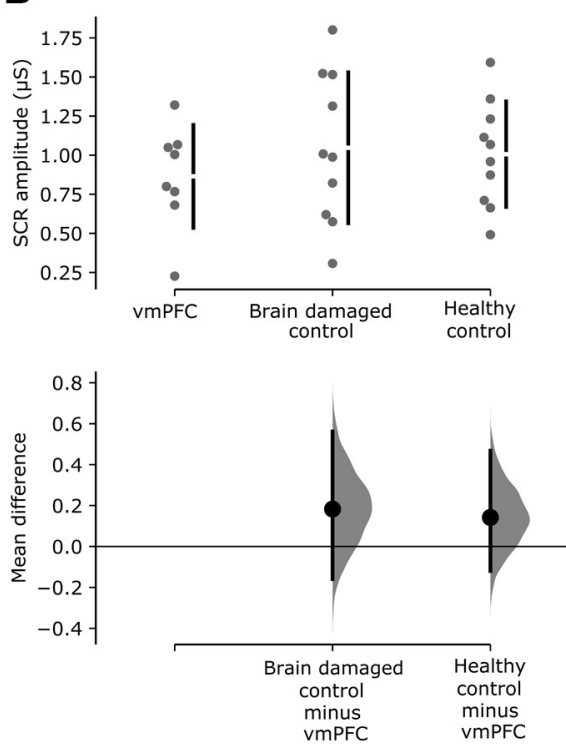

C
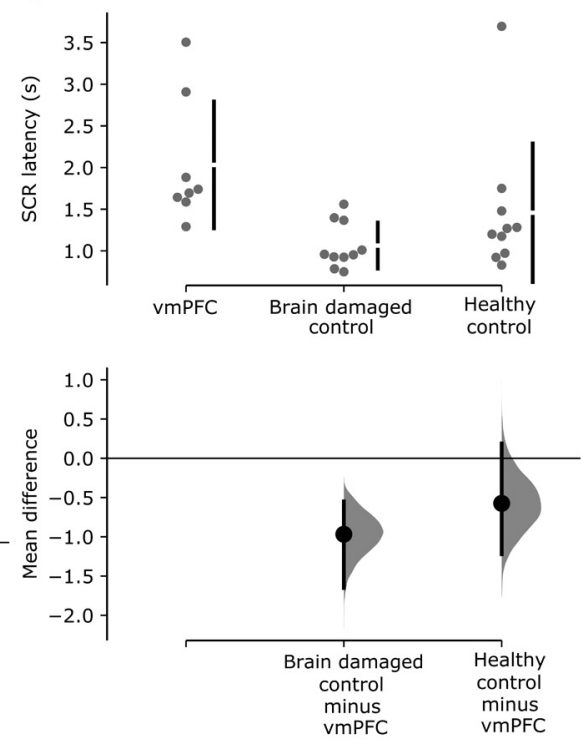

Figure 4. US intensity, SCR amplitude, and latency to US. $\boldsymbol{A}-\boldsymbol{C}$, Cumming estimation plots showing raw data and mean difference of US intensity (A), SCR amplitude to the US (B), and SCR latency to the US ( $C$ ) for the brain damaged control group and the healthy control group against the vmPFC group. On the top axes, 95\% confidence intervals are indicated by vertical error bars and group means are depicted as white gaps between the bars. On the lower axes, $95 \%$ confidence intervals are indicated by vertical error bars and mean differences, plotted as a bootstrap sampling distribution (5000 samples), are depicted as dots.

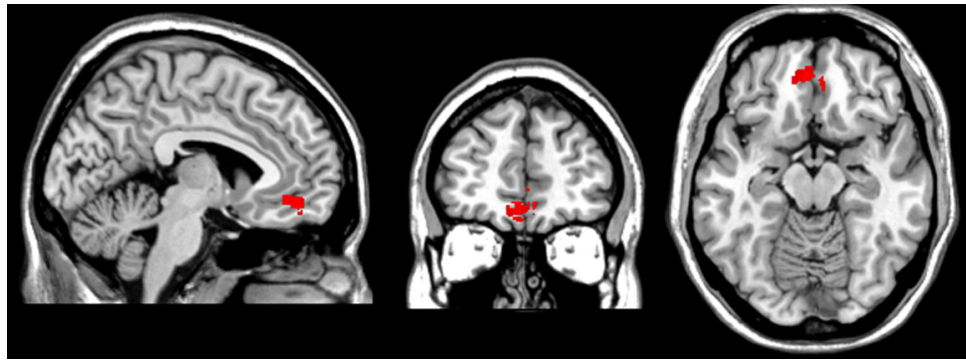

Figure 5. Results for the voxel-based lesion-symptom mapping analysis. The image shows the location of the cluster of lesioned voxels associated with reduced differential SCR during threat acquisition (MNI coordinates: $-5,47,-14$ ).

contrast, vmPFC patients did not differ significantly from HCs (mean $=1.46 \mathrm{~s}, p=0.186$ ), although the observation of Cumming estimation plots suggests that such a result may have been driven by one HC participant with a mean latency of $3.5 \mathrm{~s}$. BDCs did not differ from HCs $(p=0.397)$. These results suggest that a lesion to vmPFC does not affect the amplitude of unconditioned response, but partly affects its latency. Following this, we repeated the analysis on the acquisition data inserting the SCR latency to the US as a covariate. Crucially, this did not change the acquisition results (all covariates, $p \geq 0.768$ ).

\section{Explicit CS-US contingency awareness}

Finally, we tested whether groups differed in the awareness of CS-US contingency, as this may play a role in the acquisition of conditioned responses assessed with SCR (LaBar et al., 2004; Sevenster et al., 2014). The $\chi^{2}$ test showed no differences in the frequency of correct and incorrect responses to either $\mathrm{CS}^{+}$ $\left(\chi^{2}(2)=0.085, p=0.958\right)$ or $\mathrm{CS}^{-}\left(\chi^{2}(2)=0.49, p=0.782\right)$ among the three groups. In the $\mathrm{HC}$ and $\mathrm{BDC}$ groups, 8 of 10 participants correctly identified the $\mathrm{CS}^{+}$outcome association, while 6 of 6 participants did so in the vmPFC group. Also, 7 of 10 healthy participants, and 6 of $10 \mathrm{BDC}$ and vmPFC participants correctly identified the $\mathrm{CS}^{-}$outcome association.
Voxel-based lesion symptom mapping analysis

A cluster of 1364 voxels centered at MNI coordinates $-5,47,-14$ (maximum BM $z$-score, 3.39), in BA 11, was associated with reduced differential SCR during threat acquisition (Fig. 5). Thus, this analysis corroborates the interpretation of the causal role of vmPFC in the acquisition of pavlovian threat learning.

\section{Discussion}

A naturally occurring lesion centered on the vmPFC, and involving adjacent perigenual tissue, compromised conditioned responses during threat acquisition. This was evidenced by impaired anticipatory differential SCR amplitude between $\mathrm{CS}^{+}$and $\mathrm{CS}^{-}$in vmPFC patients compared with healthy participants and brain damaged control patients, which was also selectively correlated with damage in a cluster of voxels located in BA 11. In contrast, there was no evidence that a lesion centered on the vmPFC compromised unconditioned response amplitudes or declarative memory for CSUS contingencies. Additionally, although a lesion centered on the vmPFC partly affected unconditioned response latency, the latter did not affect conditioned response amplitude. Thus, the impairment in threat acquisition of vmPFC patients was independent of the latency difference in the unconditioned response.

The finding that a bilateral lesion centered on the vmPFC impairs conditioned physiological responses during the acquisition of threat conditioning calls for a revaluation of the role of vmPFC in pavlovian conditioning in humans. In contrast with the findings of Bechara et al. (1999), our results indicate that, in humans, the vmPFC is necessary since the early stages of threat conditioning, extending recent neuroimaging evidence that shows activity in this region during threat acquisition (Fullana et al., 2016; Dunsmoor et al., 2019). Thus, in addition to being 
involved in the extinction and reversal of previously acquired learning (Morris and Dolan, 2004; Phelps et al., 2004; Kalisch et al., 2006; Sotres-Bayon et al., 2006; Milad et al., 2007; Quirk and Mueller, 2008; Schiller et al., 2008; Schiller and Delgado, 2010; Hartley et al., 2011; Milad and Quirk, 2012; Raij et al., 2018; Dunsmoor et al., 2019; Savage et al., 2020), the vmPFC is necessary to produce sustained autonomic arousal in anticipation of threat during acquisition, which is in line with evidence in primates during reward conditioning (Rudebeck et al., 2014). Such a result seems also to support the view that the vmPFC does not exert a mere inhibitory role over amygdala activity (Koenigs et al., 2008; Hiser and Koenigs, 2018), as this should have led to heightened psychophysiological response in patients with vmPFC lesions compared with the control groups. In fact, given the role of the vmPFC in value and stimulus-outcome representation (Roesch and Schoenbaum, 2006; Schoenbaum et al., 2009, 2011; Hiser and Koenigs, 2018), model-based representations (Wilson et al., 2014), and schema-related processing and latent structure learning (Gershman and Niv, 2010; Gershman et al., 2015; Niv, 2019; Schuck et al., 2019), the impaired physiological response in vmPFC patients may suggest a causal role of this region in the acquisition of pavlovian conditioning.

Pavlovian conditioning is not simply learning about CS-US contingency (the probability of US occurrence given CS presence) or contiguity (near simultaneity of CS and US occurrence; Rescorla, 1988; McNally and Westbrook, 2006; Schultz, 2006). Rather, it involves learning about the broad structure of the task, as suggested by evidence showing the involvement of modelbased computations during pavlovian learning, occurring not just in the amygdala but also in vmPFC (Prévost et al., 2013; Pauli et al., 2019). For example, to produce a sustained conditioned response during $\mathrm{CS}^{+}$presentation, one should learn that $\mathrm{CS}^{+}$predicts US occurrence but $\mathrm{CS}^{-}$does not, and also the specific timing of US occurrence. Also, given the partial reinforcement schedule of the task, such learning cannot be acquired based solely on the previous trial but requires a representation of the long-run sequence of trials. Additionally, pavlovian learning has been suggested to involve schema-related processing and latent structure learning (Gershman and Niv, 2010; Gershman et al., 2015; Niv, 2019; Schuck et al., 2019; i.e., learning the relationship between unobservable latent causes, such as the experimental phase, and observable stimuli, such as CS and US). For example, $\mathrm{CS}^{+}$predicts US occurrence only during acquisition and not during habituation or extinction, and this structure cannot be directly observed during the task but must be inferred. Also in this case, evidence indicates a crucial role of vmPFC in these processes (Wilson et al., 2014; Chan et al., 2016; Schuck et al., 2019). Given this, the failure of patients with vmPFC lesions in producing a sustained physiological conditioned response may result from an inability to construct a detailed internal representation of the task. In the vmPFC group, the conditioned physiological response was impaired despite preserved declarative memory of CS-US contingencies, corroborating the idea that awareness of stimulus-outcome association is not sufficient to generate a conditioned physiological response in anticipation of US occurrence and that pavlovian learning is more than CSUS contingency learning. Thus, the vmPFC may be required during acquisition to turn amygdala and/or hippocampal inputs-conveying information about stimulus-outcome contingencies-into a representation of the task, which may then be translated into a sustained conditioned physiological response in anticipation of the US during $\mathrm{CS}^{+}$presentation.

The above interpretation suggests that in humans the vmPFC and adjacent perigenual tissue may have a more prominent role in threat conditioning than in rodents, where a lesion of the infralimbic mPFC (putative homolog of human vmPFC) does not impair threat acquisition (Morgan et al., 1993; Morgan and LeDoux, 1995; Morrow et al., 1999; Quirk et al., 2000; Lebrón et al., 2004). In fact, in rodents, the infralimbic mPFC appears necessary for the extinction of conditioned responses (for review, see Sotres-Bayon et al., 2006; Milad and Quirk, 2012; Orsini and Maren, 2012; Duvarci and Pare, 2014), and, in particular, it may be causally involved in the formation of the extinction memory rather than in its retrieval (Do-Monte et al., 2015). Possibly, in humans, the vmPFC may have an analogous role also during the acquisition of threat conditioning.

The current results open the doors to further research aimed at clarifying the role of $\mathrm{vmPFC}$ in threat conditioning acquisition. Given the permanent nature of the lesion, we cannot exclude a second mechanistic interpretation for the impairment of vmPFC patients during acquisition. Namely, the vmPFC may be necessary for the expression of conditioned responses, rather than acquisition per se. To disentangle these interpretations, future studies may temporarily disrupt vmPFC activity through neurostimulation during acquisition. The appearance of a conditioned response in a subsequent threat recovery test-during which vmPFC activity will be restored-would indicate that the vmPFC is necessary for the expression of conditioning. On the contrary, lack of such a response would indicate the causal role of vmPFC in threat acquisition. In addition, neuroimaging studies show greater activity of the vmPFC in response to $\mathrm{CS}^{-}$than to $\mathrm{CS}^{+}$, possibly indicating that the vmPFC processes a safety response during presentation of $\mathrm{CS}^{-}$(Fullana et al., 2016; Dunsmoor et al., 2019). Future studies may further explore the specific contribution of this region to processing safety or threat or their relative value. Also, given the extended maturational trajectory of vmPFC over the lifespan (Gogtay et al., 2004) and the age of our participants, it could be wondered whether a lesion to vmPFC at an earlier developmental stage also disrupts threat acquisition. Several studies have shown that the quality of threat conditioning acquisition changes over the course of development (Hartley and Lee, 2015); nevertheless, the extent of vmPFC involvement in these changes remains unknown. Additionally, the lesions of vmPFC patients also involved white matter tracts, including the uncinate fasciculus, which connects frontal areas with limbic areas of the temporal lobe. Thus, the extent to which damage to the white matter tracts may contribute to the current results should also be investigated. Finally, the current results also represent the opportunity for a reinterpretation of the deficit of patients with vmPFC lesion in the Iowa Gambling Task (Bechara et al., 1996, 1999; Fellows and Farah, 2005) in terms of an acquisition deficit. More broadly, they may suggest that such patients may have difficulties in following information and rules, because of an inability to acquire such rules, rather than an inability to flexibly conform their behavior according to the current set of rules.

When our results are considered together with results of patients with bilateral amygdala and hippocampus lesions (Bechara et al., 1995), they seem to indicate that the amygdala is necessary but not sufficient to generate conditioned skin conductance response, while the hippocampus is not only necessary but may also be sufficient for declarative memory of CS-US contingencies. Additionally, the preserved unconditioned response (amplitude SCR to US) and declarative memory (explicit awareness) of stimulus-outcome contingencies suggest that these two processes are not sufficient to generate conditioned physiological responses, which is in keeping with the literature on patients 
with hippocampal lesions (Bechara et al., 1995), and that they may not rely on a functional vmPFC.

In conclusion, we found that a naturally occurring bilateral lesion centered on the vmPFC compromises the generation of a conditioned psychophysiological response during the acquisition of pavlovian threat conditioning. Crucially, this result advances knowledge on the neural bases of threat conditioning, and on reinforcement learning more broadly, suggesting that the vmPFC plays a causal role in the acquisition of new learning and not just in the extinction or reversal of previously acquired learning, as previously thought. From a clinical perspective, it suggests that part of the cognitive and behavioral difficulties observed in patients with a vmPFC lesion may result from such an acquisition deficit.

\section{References}

Battaglia S, Garofalo S, di Pellegrino G (2018) Context-dependent extinction of threat memories: influences of healthy aging. Sci Rep 8:12592.

Bechara A, Tranel D, Damasio H, Adolphs R, Rockland C, Damasio AR (1995) Double dissociation of conditioning and declarative knowledge relative to the amygdala and hippocampus in humans. Science 269:11151118.

Bechara A, Tranel D, Damasio H, Damasio AR (1996) Failure to respond autonomically to anticipated future outcomes following damage to prefrontal cortex. Cereb Cortex 6:215-225.

Bechara A, Damasio H, Damasio AR, Lee GP (1999) Different contributions of the human amygdala and ventromedial prefrontal cortex to decisionmaking. J Neurosci 19:5473-5481.

Brunner E, Munzel U (2000) The nonparametric Behrens-Fisher problem: asymptotic theory and a small-sample approximation. Biom J 42:17-25.

Bukalo O, Pinard CR, Silverstein S, Brehm C, Hartley ND, Whittle N, Colacicco G, Busch E, Patel S, Singewald N, Holmes A (2015) Prefrontal inputs to the amygdala instruct fear extinction memory formation. Sci Adv 1:e1500251.

Bush G, Luu P, Posner MI (2000) Cognitive and emotional influences in anterior cingulate cortex. Trends Cogn Sci 4:215-222.

Caffarra P, Vezzadini G, Dieci F, Zonato F, Venneri A (2002) A short version of the Stroop test: normative data in an Italian population sample. Nuova Riv Neurol 12:111-115.

Capitani E, Laiacona M (1988) Aging and psychometric diagnosis of intellectual impairment: some considerations on test scores and their use. Dev Neuropsychol 4:325-330.

Chan SCY, Niv Y, Norman KA (2016) A probability distribution over latent causes, in the orbitofrontal cortex. J Neurosci 36:7817-7828.

Culbertson WC, Zillmer EA (1999) Tower of London Drexel University (TOL DX): examiner's manual. North Tonawanda, NY Multi-Health Systems.

Delgado MR, Beer JS, Fellows LK, Huettel SA, Platt ML, Quirk GJ, Schiller D (2016) Viewpoints: dialogues on the functional role of the ventromedial prefrontal cortex. Nat Neurosci 19:1545-1552.

Do-Monte FH, Manzano-Nieves G, Quiñones-Laracuente K, Ramos-Medina L, Quirk GJ (2015) Revisiting the role of infralimbic cortex in fear extinction with optogenetics. J Neurosci 35:3607-3615.

Dunsmoor JE, Kroes MCW, Li J, Daw ND, Simpson HB, Phelps EA (2019) Role of human ventromedial prefrontal cortex in learning and recall of enhanced extinction. J Neurosci 39:3264-3276.

Duvarci S, Pare D (2014) Amygdala microcircuits controlling learned fear. Neuron 82:966-980.

Fellows LK, Farah MJ (2005) Different underlying impairments in decisionmaking following ventromedial and dorsolateral frontal lobe damage in humans. Cereb Cortex 15:58-63.

Fullana MA, Harrison BJ, Soriano-Mas C, Vervliet B, Cardoner N, ÀvilaParcet A, Radua J (2016) Neural signatures of human fear conditioning: an updated and extended meta-analysis of fMRI studies. Mol Psychiatry 21:500-508.

Gershman SJ, Niv Y (2010) Learning latent structure: carving nature at its joints. Curr Opin Neurobiol 20:251-256.

Gershman SJ, Norman KA, Niv Y (2015) Discovering latent causes in reinforcement learning. Curr Opin Behav Sci 5:43-50.
Gläscher J, Rudrauf D, Colom R, Paul LK, Tranel D, Damasio H, Adolphs R (2010) Distributed neural system for general intelligence revealed by lesion mapping. Proc Natl Acad Sci U S A 107:4705-4709.

Gogtay N, Giedd JN, Lusk L, Hayashi KM, Greenstein D, Vaituzis AC, Nugent TF, Herman DH, Clasen LS, Toga AW, Rapoport JL, Thompson PM (2004) Dynamic mapping of human cortical development during childhood through early adulthood. Proc Natl Acad Sci U S A 101:81748179 .

Harrison BJ, Fullana MA, Via E, Soriano-Mas C, Vervliet B, MartínezZalacaín I, Pujol J, Davey CG, Kircher T, Straube B, Cardoner N (2017) Human ventromedial prefrontal cortex and the positive affective processing of safety signals. Neuroimage 152:12-18.

Hartley CA, Lee FS (2015) Sensitive periods in affective development: nonlinear maturation of fear learning. Neuropsychopharmacology 40:50-60.

Hartley CA, Fischl B, Phelps EA (2011) Brain structure correlates of individual differences in the acquisition and inhibition of conditioned fear. Cereb Cortex 21:1954-1962.

Hiser J, Koenigs M (2018) The multifaceted role of the ventromedial prefrontal cortex in emotion, decision making, social cognition, and psychopathology. Biol Psychiatry 83:638-647.

Ho J, Tumkaya T, Aryal S, Choi H, Claridge-Chang A (2019) Moving beyond P values: data analysis with estimation graphics. Nat Methods 16:565-566.

Holmes CJ, Hoge R, Collins L, Woods R, Toga AW, Evans AC (1998) Enhancement of MR images using registration for signal averaging. J Comput Assist Tomogr 22:324-333.

Kalisch R, Korenfeld E, Stephan KE, Weiskopf N, Seymour B, Dolan RJ (2006) Context-dependent human extinction memory is mediated by a ventromedial prefrontal and hippocampal network. J Neurosci 26:95039511.

Kennedy C, Zillmer E, Culbertson W (2000) Assessing executive function in the MR population: an investigation of the TOL-Dx and MWCST. Arch Clin Neuropsychol 19:661-671.

Koenigs M, Tranel DT, Damasio AR (2007) The Lesion method in cognitive neuroscience. In: Handbook of psychophysiology (Cacioppo JT, Tassinary LG, Berntson GG, eds), pp 139-156. Cambridge, UK: Cambridge UP.

Koenigs M, Huey ED, Raymont V, Cheon B, Solomon J, Wassermann EM, Grafman J (2008) Focal brain damage protects against post-traumatic stress disorder in combat veterans. Nat Neurosci 11:232-237.

LaBar KS, Cook CA, Torpey DC, Welsh-Bohmer KA (2004) Impact of healthy aging on awareness and fear conditioning. Behav Neurosci 118:905-915.

Lebrón K, Milad MR, Quirk GJ (2004) Delayed recall of fear extinction in rats with lesions of ventral medial prefrontal cortex. Learn Mem 11:544-548.

Lykken DT (1972) Range correction applied to heart rate and to GSR data. Psychophysiology 9:373-379.

McNally GP, Westbrook RF (2006) Predicting danger: the nature, consequences, and neural mechanisms of predictive fear learning. Learn Mem 13:245-253.

Milad MR, Quirk GJ (2012) Fear extinction as a model for translational neuroscience: ten years of progress. Annu Rev Psychol 63:129-151.

Milad MR, Wright CI, Orr SP, Pitman RK, Quirk GJ, Rauch SL (2007) Recall of fear extinction in humans activates the ventromedial prefrontal cortex and hippocampus in concert. Biol Psychiatry 62:446-454.

Morgan MA, LeDoux JE (1995) Differential contribution of dorsal and ventral medial prefrontal cortex to the acquisition and extinction of conditioned fear in rats. Behav Neurosci 109:681-688.

Morgan MA, Romanski LM, LeDoux JE (1993) Extinction of emotional learning: contribution of medial prefrontal cortex. Neurosci Lett 163:109-113.

Morris J, Dolan R (2004) Dissociable amygdala and orbitofrontal responses during reversal fear conditioning. Neuroimage 22:372-380.

Morrow BA, Elsworth JD, Rasmusson AM, Roth RH (1999) The role of mesoprefrontal dopamine neurons in the acquisition and expression of conditioned fear in the rat. Neuroscience 92:553-564.

Niv Y (2019) Learning task-state representations. Nat Neurosci 22:15441553.

Novelli G, Papagno C, Capitani E, Laiacona M, Cappa SF, Vallar G (1986) Tre test clinici di memoria verbale a lungo termine. Taratura su soggetti normali. Arch Psicol Neurol Psichiatr 47:278-296.

Orsini A, Grossi D, Capitani E, Laiacona M, Papagno C, Vallar G (1987) Verbal and spatial immediate memory span: normative data from 1355 adults and 1112 children. Ital J Neurol Sci 8:539-548. 
Orsini CA, Maren S (2012) Neural and cellular mechanisms of fear and extinction memory formation. Neurosci Biobehav Rev 36:1773-1802.

Pauli WM, Gentile G, Collette S, Tyszka JM, O’Doherty JP (2019) Evidence for model-based encoding of Pavlovian contingencies in the human brain. Nat Commun 10:1099.

Phelps E, Delgado MR, Nearing K, Joseph L (2004) Extinction learning in humans: role of the amygdala and vmPFC. Neuron 43:897-905.

Prévost C, McNamee D, Jessup RK, Bossaerts P, O’Doherty JP (2013) Evidence for model-based computations in the human amygdala during Pavlovian conditioning. PLoS Comput Biol 9:e1002918.

Quirk GJ, Mueller D (2008) Neural mechanisms of extinction learning and retrieval. Neuropsychopharmacology 33:56-72.

Quirk GJ, Russo GK, Barron JL, Lebron K (2000) The role of ventromedial prefrontal cortex in the recovery of extinguished fear. J Neurosci 20:6225-6231.

Raij T, Nummenmaa A, Marin M-F, Porter D, Furtak S, Setsompop K, Milad MR (2018) Prefrontal cortex stimulation enhances fear extinction memory in humans. Biol Psychiatry 84:129-137.

Rescorla RA (1988) Pavlovian conditioning: it's not what you think it is. Am Psychol 43:151-160.

Rescorla RA, Wagner AR (1972) A theory of Pavlovian conditioning: variations in the effectiveness of reinforcement and nonreinforcement BT. In: Classical conditioning. II, current research and theory (Black $\mathrm{AH}$, Prokasy WF, eds), pp 64-99. New York: Appleton-Century-Crofts.

Roesch M, Schoenbaum G (2006) From associations to expectancies: orbitofrontal cortex as gateway between the limbic system and representational memory. In: The orbitofrontal cortex (Zald DH, Rauch SL, eds), pp 199236. Oxford, UK: Oxford UP.

Rorden C, Brett M (2000) Stereotaxic display of brain lesions. Behav Neurol 12:191-200.

Rorden C, Bonilha L, Nichols TE (2007) Rank-order versus mean based statistics for neuroimaging. Neuroimage 35:1531-1537.

Rudebeck PH, Putnam PT, Daniels TE, Yang T, Mitz AR, Rhodes SV, Murray EA (2014) A role for primate subgenual cingulate cortex in sustaining autonomic arousal. Proc Natl Acad Sci U S A 111:5391-5396.
Savage HS, Davey CG, Fullana MA, Harrison BJ (2020) Clarifying the neural substrates of threat and safety reversal learning in humans. Neuroimage 207:116427.

Schiller D, Delgado MR (2010) Overlapping neural systems mediating extinction, reversal and regulation of fear. Trends Cogn Sci 14:268-276.

Schiller D, Levy I, Niv Y, LeDoux JE, Phelps EA (2008) From fear to safety and back: reversal of fear in the human brain. J Neurosci 28:11517-11525.

Schoenbaum G, Roesch MR, Stalnaker TA, Takahashi YK (2009) A new perspective on the role of the orbitofrontal cortex in adaptive behaviour. Nat Rev Neurosci 10:885-892.

Schoenbaum G, Takahashi Y, Liu T-L, McDannald MA (2011) Does the orbitofrontal cortex signal value? Ann N Y Acad Sci 1239:87-99.

Schuck NW, Wilson R, Niv Y (2019) A state representation for reinforcement learning and decision-making in the orbitofrontal cortex. In: Goaldirected decision making (Morris R, Bornstein A, Shenhav A, eds), pp 259-278. Cambridge, MA: Academic.

Schultz W (2006) Behavioral theories and the neurophysiology of reward. Annu Rev Psychol 57:87-115.

Sevenster D, Beckers T, Kindt M (2014) Fear conditioning of SCR but not the startle reflex requires conscious discrimination of threat and safety. Front Behav Neurosci 8:1-9.

Siddle DAT, Bond NW, Packer JS (1988) Comparator theories of habituation: a comment on Mackintosh's analysis. Biol Psychol 27:59-63.

Sjouwerman R, Lonsdorf TB (2019) Latency of skin conductance responses across stimulus modalities. Psychophysiology 56:e13307.

Sotres-Bayon F, Cain CK, LeDoux JE (2006) Brain mechanisms of fear extinction: historical perspectives on the contribution of prefrontal cortex. Biol Psychiatry 60:329-336.

Spinnler H, Tognoni G (1987) Standardizzazione e taratura italiana di test neuropsicologici. Gruppo Italiano per lo Studio Neuropsicologico dell'Invecchiamento. Ital J Neurol Sci 8:111-119.

Sutton RS (1988) Learning to predict by the methods of temporal differences. Mach Learn 3:9-44.

Wilson RC, Takahashi YK, Schoenbaum G, Niv Y (2014) Orbitofrontal cortex as a cognitive map of task space. Neuron 81:267-279. 